\title{
Polypoid adenocarcinoma in the efferent jejunal loop after total gastrectomy in a Muir-Torre syndrome patient
}

\author{
Jaime Ruiz-Tovar • Javier Lacueva • Israel Oliver • \\ Amparo Martinez-Blasco $\cdot$ Rafael Calpena
}

Accepted: 4 October 2011 /Published online: 18 October 2011

(C) Springer-Verlag 2011

\section{Dear Editor:}

Once no loss of expression and no germline mutations of genes MLH1, MSH2, MSH6, and PMS2 were found, additional molecular analysis were done. Extensive deletions and insertions were also ruled out, but a compound biallelic MYH germline mutation (Y165C) was found. Moreover, a subcutaneous lesion in the forehead was removed and diagnosed of sebaceous adenoma. In summary, along a 15-year period, our patient developed one gastric adenocarcinoma, three colonic adenocarcinomas as well as several tubulo-villous adenomas, one small bowel adenocarcinoma, and one sebaceous adenoma. This clinical picture has led us up to the diagnosis of Muir-Torre syndrome (MTS).

MTS is characterized by the association of sebaceous gland neoplasms or keratoacanthomas with visceral malignant diseases, most frequently colorectal carcinomas but also endometrial and urological neoplasms. Other neoplasms such as upper gastrointestinal carcinomas are also found but they develop more rarely. MTS is considered a clinical variant of hereditary non-polyposis colorectal cancer (HNPCC), and up to two thirds of MTS patients present germline mutations in HNPCC genes, predominantly in the MSH2 gene. In addition, other genetic alterations such as MYH germline mutations have also been described.

J. Ruiz-Tovar · J. Lacueva · I. Oliver · A. Martinez-Blasco •

R. Calpena

Department of General and Digestive Surgery,

General University Hospital Elche,

Alicante, Spain

J. Ruiz-Tovar $(\bowtie)$

Corazon de Maria, 64, $7^{\circ} \mathrm{J}$,

28002 Madrid, Spain

e-mail: jruiztovar@gmail.com
The report of a polypoid small bowel adenocarcinoma arising in the efferent with jejunal loop, after a total gastrectomy performed 15 years before is exceptional. Just one case of synchronous jejunal carcinoma has been reported in a patient with an MYH-associated polyposis (MAP) syndrome. The full clinical spectrum of the recently described MAP syndrome is variable and yet to be elucidated. It often appears clinically similar to attenuated familial adenomatous polyposis (FAP), but some individuals may show a phenotype that overlaps from FAP to HNPCC syndrome, as it is shown in our patient. Small bowel carcinomas in MTS patients have been very rarely reported to date, most of them located at the duodenum.

Whether it is beneficial or not to screen these patients for small bowel neoplasias is still unknown. The risk of small bowel cancer in HNPCC patients is estimated to be around $4 \%$, and the localization is almost similar in the duodenum and the jejunum, but less frequent in the ileum. However, screening for small bowel cancer in HNPCC patients has not been included yet in the guidelines for surveillance, partly due to the fact that the possibility to explore the small bowel was limited until recently. Up to $25 \%$ of patients with MAP syndrome may present duodenal adenomatous polyposis and have shown to have a high relative risk of duodenal cancer, but the incidence of small bowel cancer is unknown in these patients.

In the HNPCC and the MTS patients, the behavior of the visceral malignant diseases seems to be less aggressive and these patients tend to survive longer than their sporadic counterparts, so the prognosis might be improved by an early diagnosis. Then, a screening by a noninvasive procedure such as video capsule endoscopy may be advisable, although further studies are needed to show the real incidence of small bowel lesions in these patients. 